\title{
Reviewer Acknowledgements for Global Journal of Health Science, Vol. 8, No. 12
}

Global Journal of Health Science wishes to acknowledge the following individuals for their assistance with peer review of manuscripts for this issue. Their help and contributions in maintaining the quality of the journal are greatly appreciated.

Global Journal of Health Science is recruiting reviewers for the journal. If you are interested in becoming a reviewer, we welcome you to join us. Please find the application form and details at http://recruitment.ccsenet.org and e-mail the completed application form to gjhs@ccsenet.org.

\section{Reviewers for Volume 8, Number 12}

Ahmet Büber

Akça Toprak Ergönen

Alexandrina L. Dumitrescu

Amir Hassani

Ayşegül Özdemir Topaloğlu

Barry E. Chatterton

Benjamin Holfelder

Brahim Hadji

Damien Offner

Davide Sartini

Ehsan Homaei

Francesco Teodori

Franciszek Burdan

Gabriele Messina

Genna M. Mulvey

Giancarlo Cicolini

Helmy A. Heba

Houman Manoochehri

Jereme D. Wilroy

Jesús Álvarez-Herms

Joanna Pawlak

Kay LH Wu

L. Dossche

Lászlpó Góth
Li Ya Lin

Lobna A. Aly

Lucio P. Neves

Mahbobeh Faramarzi

Marcelo Sperandio São Leopoldo Mandic

Medhat K. El-Shazly

Michael H. Humphreys

Michele Lanza

Mohamed Khalifa

Murat Eroglu

Natalia Lazzarin

Paola Di Giulio

Professor E. Vlahaki

Raildo Coqueiro

Rungrote Natesirinilkul

Saori I. Braun

Saqib A.K. Utman

Sharynn Schuster

Sonia Julià-Sánchez

sonia sahli

Thammanard Charernboon

Vaner Köksal

Wen-Juan Wang

Yee Chang Soh 